\title{
Bioweathering of metal-bearing phases by siderophore producing bacteria Pseudomonas fluorescens
}

\section{ANNA POTYSZ}

\author{
University of Wroclaw
}

Presenting Author: anna.potysz@uwr.edu.pl

The large volumes of metallurgical wastes being produced raise environmental concerns. Bacterially-mediated weathering may lead to release of metals into the environment, leaving an irreversible fingerprint on the solid surface. However, the extent of (bio)weathering depends on many physico-chemical and biotic factors involved.

The dissolution of lead matte and copper slag was investigated to decipher (bio)dissolution of metal-bearing phases being important components of studied industrial wastes. Lead matte and copper slag were subjected to (bio)weathering in a semiopen pass flow through experiments under conditions involving bacteria Pseudomonas fluorescens (strain DSM 50091) versus abiotic growth medium and ultrapure water. The risk of metal mobilization was assessed by means of (bio)leaching (quantification by ICP-MS). Leachate digestion allowed to determine the amount of metals associated with biomass. The weathering of the phase surface was identified by scanning electron microscopy (SEM-EDS). The siderophore detection was done by recording of optical spectra at the wavelength range $300-600 \mathrm{~nm}$, whereas siderophore concentration was determined by measuring absorbance at $405 \mathrm{~nm}$ following calculations according to the Beer Lambert law. Geochemical modelling was used to determine the degree of saturation of the leaching solutions with respect to possible secondary precipitates.

A long-term waste exposure to $P$. fluorescens revealed an important release of metals, indicating that bacterially mediated weathering enhances dissolution of industrial wastes relative to abiotic conditions. Excretion of siderophores varied depending on the type of waste subjected to bioweathering experiments proving that the wastes affected bacterial activity to a different extent. This study has important implication for the landfill environments where a long-term disposal of metallurgical wastes may continue to be a source of metal-laden leachates.

Acknowledgements: This work was financially supported by the National Science Centre ( $\mathrm{NCN})$ in Poland in the frame of SONATA program under grant agreement UMO2018/31/D/ST10/00738 to AP. 